\title{
Evaluación calórica y sensorial de una mezcla para helado formulado con inulina como sustituto parcial de grasa
}

\section{Sensory and calorie evaluation of an ice cream mix, where inulin is incorporated as a partial substitute for fat}

\author{
Jorge Eliécer Rodríguez-Ordóñez ; Luis Fernando Mejía-Giraldo ${ }^{1}$; Liliana Serna-Cock ${ }^{3 *}$
}

1Ing. Agroindustrial, Meals de Colombia S.A.S., Armenia, Quindío, Colombia. e-mail: jerodriguezo@unal.edu.co; Dhttps://orcid.org/0000-0002-6446-8943

${ }^{2}$ Ing. Agroindustrial. Universidad de San Buenaventura. Cali, Valle del Cauca, Colombia. e-mail: Ifmejia@usbcali.edu.co; Dhttps://orcid.org/0000-0002-62916191

${ }^{3}$ Bacterióloga, Ph.D. Universidad Nacional de Colombia, Sede Palmira. Palmira,Valle del Cauca, Colombia. e-mail: Iserna@unal.edu.co; Dhttps://orcid. org/0000-0003-2911-0871

*autor de correspondencia: Iserna@unal.edu.co

Cómo citar: Rodríguez-Ordóñez, J.E.; Mejía-Giraldo, L.F.; Serna-Cock, L. 2019. Evaluación calórica y sensorial de una mezcla para helado formulado con inulina como sustituto parcial de grasa. Rev. U.D.C.A Act. \& Div. Cient. 22(2):e1294. http://doi.org/10.31910/ rudca.v22.n2.2019.1294

Artículo de acceso abierto publicado por Revista U.D.C.A Actualidad \& Divulgación Científica, bajo una licencia Creative Commons CC BY-NC 4.0

Recibido: Noviembre 30 de 2017

Aceptado: Julio 15 de 2019

Editado por: Ingeborg Zenner de Polanía

\section{RESUMEN}

Se evaluó el aporte calórico, el contenido de grasa saturada y la aceptación sensorial de helados de vainilla formulados con inulina, como sustituto parcial de grasa saturada. Se formularon por triplicado cuatro mezclas para helados, en las cuales, se reemplazó parte de la grasa de palma (2, 3 y 4\%) por inulina comercial. La grasa del tratamiento control, se formuló con 7\% de grasa vegetal saturada. Para el reemplazo, se utilizó una relación 1:0,25 (grasa saturada:inulina). El aporte calórico, se calculó mediante la medición de energía bruta. El análisis sensorial, se realizó con 23 jueces consumidores habituales de helado. Se concluyó que en la formulación de helados de vainilla es posible reemplazar el $57,14 \%$ de grasa vegetal saturada por inulina sin que se afecten las propiedades sensoriales en el producto, logrando reducir en un 16\%, el contenido calórico y, en 48,6\%, el contenido de grasa saturada. Por lo tanto, la sustitución de grasa vegetal por inulina es una alternativa, que permite ofrecer en el mercado helados funcionales, con bajo contenido calórico, sin perder la palatabilidad, que hace a los helados un producto placentero para la gran mayoría de la población mundial.

Palabras clave: prebiótico; oligofructanos; caloría; grasa de palma; propiedades organolépticas

\section{ABSTRACT}

The caloric intake, saturated fat content and sensory acceptance of vanilla ice cream formulated with inulin as a partial substitute for saturated fat was evaluated. Ice cream mixtures by triplicate were formulated, and part of the palm fat (2,3 and 4\%) was replaced by commercial inulin. The fat of the control treatment was formulated with $7 \%$ vegetable fat. For the replacement of fat, a ratio of 1 : 0.25 (fat: inulin) was used. The caloric intake was calculated by measuring gross energy. The sensory analysis was carried out with 23 habitual consumers judges of ice cream. It was concluded that in the formulation of vanilla ice cream is possible to replace $57.14 \%$ vegetable fat for inulin without affecting the sensory properties in 
the product, with this is possible to obtain $16 \%$ reduction in the caloric content and $48.6 \%$ in the content of saturated fat. Therefore, the replacement of vegetable fat by inulin is an alternative that allows offering functional ice cream with low caloric content, without losing the palatability that makes ice cream pleasant for most of the world population.

Keywords: prebiotic; oligofructans; calorie; palm fat; organoleptic properties.

\section{INTRODUCCIÓN}

El helado ha sido parte de las culturas oriental y occidental y es el postre favorito de muchas personas, a nivel mundial. En Colombia, se consumen 3,1L de helado al año por persona; este consumo es bajo comparado con el mayor consumidor de helados en el mundo, Nueva Zelanda, que consume 26,3L/año por persona; no obstante, entre 2012 y 2017, el mercado de helados creció $17,4 \%$, con ventas en 2017, que alcanzaron más de 111 millones de dólares, demostrando que el negocio va en crecimiento sostenido (Portafolio, 2019). En Colombia, más de la mitad de la población $(56 \%)$ está por encima de su peso ideal (ENSIN, 2015). El aumento en el consumo de helado en Colombia, se podría considerar como uno de los factores que explican el sobrepeso en la población colombiana. Según la normatividad colombiana (NTC 1239), un helado es un producto edulcorado obtenido de una emulsión de grasa y proteínas o de agua y aditivos (ICONTEC, 2002). Debido al aporte calórico que representa el consumo de helado, se han realizado investigaciones tendientes a reducir el contenido de grasa en los helados emulsificados; sin embargo, la reducción de grasa provoca cambios sensoriales y texturales indeseables (Akbari et al. 2016; Akbaria et al. 2019).

Es difícil promediar el aporte calórico por litro de un helado, ya que las cifras que se reportan en la literatura corresponden a promedios de formulaciones diferentes, las cuales, incluyen los productos conocidos como nieves, que son en base agua y no contienen grasa; sin embargo, para ilustrar el valor calórico de los helados emulsificados, se presentan la referencia Mágnum almendras de Unilever, un producto líder en Latinoamérica, que aporta $272 \mathrm{Kcal} / \mathrm{kg}$ (Unilever, 2016) y un cono de helado de nata y nueces, que aporta 286 calorías por 100g; estas calorías corresponden al 13,6 y 14,3, respectivamente, de la energía necesaria en una dieta de 2000 calorías día. El 16\% del aporte calórico de estos helados proviene de grasa láctea y no láctea (Marshall et al. 2003).

La grasa en los helados, se puede reducir mediante el reemplazo total o parcial con ingredientes de menor energía (Akbaria et al. 2019). Los sustitutos de grasas son sustancias de origen lipídico, glúcido o combinaciones de estas, que pueden imitar las propiedades físicas y sensoriales de la grasa, con menor aporte de calorías (Zoulias et al. 2002). Para sustituir la grasa en helados, se han utilizado maltodextrinas y polidextrosa, los cuales, tienen la ventaja de producir un mínimo de efectos negativos en la producción, vida útil y precio del helado (Goff \& Hartel, 2013). Para el mismo fin, se han utilizado productos comerciales, como Simplesse ${ }^{\circledR} 100$, que es un polvo microparticulado que simula la grasa emulsionada y DairyLo ${ }^{\mathrm{TM}}$, que es un concentrado de proteína de suero modificado, que puede formar una red similar a un gel (Yazici \& Akgun, 2004). Recientemente, se han empleado hidrolizados de proteína de soya como un sustituto de la grasa y se ha reportado que, en helados, donde se sustituyó el 50\% de la grasa por hidrolizados de proteína de soya y goma xantana en proporción $96: 4$, se presentaron propiedades sensoriales y de textura similares a las del helado de control, que contenían 10\% de grasa (Liu et al. 2018).

La inulina es particularmente adecuada para el reemplazo de grasa en helados (Franck, 2002); tiene capacidad gelificante y una estructura cremosa blanca, que se puede incorporar fácilmente a otros ingredientes, para reemplazar la grasa en un 100\% (Tiwari et al. 2015). Las compañías de alimentos, en su interés por diferenciarse de sus competidores, han encontrado un segmento de mercado, donde los consumidores valoran los alimentos que contribuyan con la salud y el bienestar; tal es el caso de los alimentos formulados con inulina (Hernández-Carranza \& Jiménez-Munguía, 2010).

La inulina es un oligofructano, compuestos que son estables a temperaturas inferiores a $140^{\circ} \mathrm{C}$, tienen sabor neutro ligeramente dulce, con poder edulcorante, que solo alcanza entre 10 y $30 \%$, respecto a la sacarosa; son estables en rangos de $\mathrm{pH}$ 4-7 en refrigeración, fácilmente hidrolizables por ácidos y enzimas (Hernández-Carranza \& Jiménez-Munguía, 2010; Mensink et al. 2015), altamente solubles en agua, ayudan en el balance de las dietas y aportan compuestos que se comportan como fibra y carbohidratos no asimilables (Barclay et al. 2012). Por lo anterior, la inulina, al igual que otros oligofructanos, son una excelente alternativa para sustituir ingredientes de alto valor calórico en la reformulación de alimentos. Adicionalmente, los oligofructanos se comportan como prebióticos, al ser promotores del desarrollo de microrganismos benéficos en el tracto digestivo (Li et al. 2015; Kolida \& Gibson, 2007).

Varias investigaciones reportan el uso de la inulina en helados, el efecto sobre la reología de la emulsión, el contenido graso y los cambios sensoriales (Akbari et al. 2016; Akalin et al. 2008; Ismail et al. 2013; Tiwari et al. 2015; Bayarri et al. 2010). Rodríguez \& Campderrós (2015) lograron obtener una formulación de helado libre de grasa incorporando inulina y almidón modificado, con características sensoriales y físicas similares a las que presentó la muestra control; sin embargo, la valoración calórica y el contenido de grasas saturadas de las formulaciones de helado no han sido reportados en la literatura científica.

El objetivo de esta investigación fue evaluar el aporte calórico, contenido de grasa saturada y la aceptación sensorial en helados de vainilla, por efecto de sustituir parcialmente la grasa por inulina.

\section{MATERIALES Y MÉTODOS}

Formulación de helado: En la formulación de helado, se utilizó por triplicado un diseño unifactorial (reemplazo de grasa saturada), con cuatro niveles, correspondientes al porcentaje de reemplazo de grasa saturada por inulina comercial $(0,2,3$, y $4 \%$ y y los tratamientos 
se nombraron como (M1), (M2), (M3), y (M4), respectivamente. Las variables de respuesta fueron contenido calórico, contenido de grasa saturada y prueba de aceptación sensorial. Los cálculos de las cuatro formulaciones, se realizaron cumpliendo la resolución 1804 de 1989 de Colombia (INVIMA, 1989) y se presentan en la tabla 1. La mezcla del tratamiento de referencia sin adición de inulina (M1), se acogió a la clasificación de helados de leche con grasa vegetal. (M1), se formuló con 7\% de grasa saturada vegetal de palma. Para el reemplazo de grasa saturada en las formulaciones, se utilizó una relación grasa saturada: inulina comercial de 1:0,25, quedando las formulaciones (M2), (M3) y (M4) con contenidos de inulina de 0,$5 ; 075$ y $1 \%$ y grasa vegetal de 5,4 y 3\%, respectivamente. Se tuvieron en cuenta dos criterios para utilizar máximo el 1\% de inulina comercial: primero, al emplear entre el 2,5 y el 5\% de inulina en la mezcla de helado, este se endurece y la velocidad para derretirse, se duplica (Ismail et al. 2013) y, el segundo criterio, fue el costo de la formulación, que afecta directamente el costo del helado, concentraciones de inulina por encima del 1\% en una formulación no podría utilizarse para formular helados económicos para consumo masivo, dado que se incrementaría en 6 veces el costo de la formulación.
Se utilizó glucosa comercial, suministrada por la empresa Ingredion S.A. Colombia, grasa vegetal saturada, adquirida en Team Colombia (referencia helados PS80), leche en polvo descremada, marca Colanta, azúcar blanco (Manuelita), vainilla Mane (ref 14059), leche líquida, marca Latti D1, estabilizante Cremodan SE 46-2 de Dúas rodas y Orafti GR de Beneo, que es inulina con pureza mayor a 90\%.

Preparación del helado: En la preparación de los helados, se llevaron a cabo las etapas de cálculos, de formulación, de pesaje de ingredientes, de disolución de la mezcla, de pasteurización, de enfriamiento, de maduración, de batido, de envasado, de endurecido y, finalmente, de almacenamiento.

Una vez realizados los cálculos de las cuatro formulaciones, los ingredientes se pesaron, utilizando balanza de precisión (Metler Toledo S6002S, Alemania). La leche, se adicionó en recipiente de acero inoxidable, con capacidad de $7 \mathrm{~L}$. Se inició el proceso de pasteurización, calentando la leche hasta $60^{\circ} \mathrm{C}$, utilizando un fogón a gas natural (Sueco, Colombia). Alcanzada la temperatura, se adicionaron los demás ingredientes, iniciando con la grasa. La mezcla, se agitó con una espátula de teflón; posteriormente, se utilizó

Tabla 1. Formulaciones para $100 \mathrm{~g}$ de mezcla para helado de vainilla. M1: tratamiento control; M2-M4: tratamientos donde se reemplazó grasa por inulina, en proporción 1:0,25.

\begin{tabular}{|c|c|c|c|c|}
\hline Material / Muestra & M1 & M2 & M3 & M4 \\
\hline Glucosa (g) & 2,00 & 2,00 & 2,00 & 2,00 \\
\hline Grasa de palma (g) & 7,00 & 5,00 & 4,00 & 3,00 \\
\hline L. polvo descremada (g) & 2,00 & 2,00 & 2,00 & 2,00 \\
\hline Azúcar blanco $(\mathrm{g})$ & 13,00 & 13,00 & 13,00 & 13,00 \\
\hline Aroma vainilla (g) & 0,10 & 0,10 & 0,10 & 0,10 \\
\hline Leche líquida entera $(\mathrm{g})$ & 75,55 & 77,05 & 77,80 & 78,55 \\
\hline Estabilizante $(\mathrm{g})$ & 0,35 & 0,35 & 0,35 & 0,35 \\
\hline Inulina $(g)$ & 0,00 & 0,50 & 0,75 & 1,00 \\
\hline
\end{tabular}

un blending eléctrico (Oster, México), durante $5 \mathrm{~min}$, a 3600rpm, para garantizar la total disolución de todos los componentes. La mezcla, se calentó nuevamente hasta $75^{\circ} \mathrm{C}$, durante $15 \mathrm{~min}$, tiempo, en el cual, se logró la pasterización de la mezcla. Para el enfriamiento, la mezcla se llevó a un baño con agua helada a $\left(2^{\circ} \mathrm{C}\right)$, hasta alcanzar en la mezcla $15^{\circ} \mathrm{C}$. Posteriormente, la mezcla se llevó a refrigeración a $4^{\circ} \mathrm{C}$; alcanzada la temperatura, se dejó la mezcla a la misma temperatura, por $4 \mathrm{~h}$ (tiempo de maduración), etapa necesaria para que la muestra sufriera transformaciones fisicoquímicas, que son básicas en la posterior etapa de batido. El batido, se realizó en un equipo auto contenido de $1 \mathrm{~L}$, con capacidad de enfriamiento de -35 y $-25^{\circ} \mathrm{C}$, con cuchilla de raspado en material fexible en forma de $\mathrm{V}$, a 45rpm (Delonghi GM 6000, China), obteniendo helado entre -4 y $-1^{\circ} \mathrm{C}$. Después, se procedió al envasado, para lo cual, se utilizaron recipientes de polietileno con tapa y, con el fin de obtener la dureza que caracteriza el helado, los tratamientos se llevaron a congelación a $-19^{\circ} \mathrm{C}$, por un tiempo mínimo de $12 \mathrm{~h}$ y máximo de $24 \mathrm{~h}$ (nevera
Haceb, Colombia). Transcurrida esta etapa, se procedió a realizar los análisis fisicoquímicos y sensoriales.

Análisis fisicoquímico: Se tomaron muestras de $250 \mathrm{~g}$ cada una y se analizaron contenido calórico y contenido de grasa saturada total.

El contenido calórico, se midió mediante cuantificación de energía bruta, utilizando la técnica factor de Atwater (Zou et al. 2007). La grasa saturada total, se cuantificó siguiendo la norma técnica AOAC 996.06 (2001).

Evaluación sensorial: Para la evaluación sensorial, se siguieron las metodologías propuestas por Dooley et al. (2010) y Guinard et al. (1996). Se utilizaron 23 personas de ambos sexos $(56,5 \%$ fueron mujeres), mayores de 18 años y hasta 45 años, trabajadores de la empresa Meals de Colombia, consumidores habituales de, por lo menos, dos veces por semana de helado de vainilla. Las 
pruebas sensoriales, se realizaron en dos etapas, inicialmente, presentando a los jueces cuatro muestras de helado de vainilla de $15 \mathrm{~g}$, aproximadamente, en vasos plásticos blancos de 4 onzas, identificados con números de tres dígitos. En el cuestionario, se registraba el nombre, la fecha, y se indicaba lo siguiente: "Pruebe las cuatro muestras de los helados que tiene en frente, empezando con la muestra de la izquierda, muestras 321, 768, 098, 432. Haga un círculo a la muestra que prefiere. Usted debe escoger una muestra, aunque no esté seguro”. Se solicitó consignar el grado de aceptación, utilizando una escala hedónica de 7 niveles, así: me gusta mucho, me gusta moderadamente, me gusta poco, no me gusta ni me disgusta, me disgusta muchísimo, me disgusta moderadamente y me disgusta mucho (categorías). Para cada una de las muestras presentadas, no se les suministró más información, para evitar posibles sesgos en las respuestas (Thompson et al. 2009). Adicionalmente, durante la evaluación sensorial, se realizó una prueba de referencia, con el objetivo de que los panelistas escogieran una muestra como la preferida entre 4 muestras; la testigo, sin adición de inulina y las muestras que contenían el 0,5\%, el 0,75\% y el 1\%.

En el análisis sensorial, las categorías se convirtieron en puntajes numéricos del 1 al 7, donde 1 significa me disgusta mucho y siete significa me gusta mucho. Los puntajes numéricos se analizaron mediante pruebas no paramétricas, utilizando el software estadístico Statgraphics Centurión XVI. Para determinar diferencias significativas entre medias, se utilizó la Prueba de Duncan, con una significancia del 95\%.

\section{RESULTADOS Y DISCUSIÓN}

Análisis fisicoquímicos: En la tabla 2, se presentan los valores promedios de tres repeticiones con las desviaciones estándar de energía y grasa saturada de helados de vainilla, formulados con inulina.

En términos de producto terminado, los resultados indican que $100 \mathrm{~g}$ de helado de vainilla formulado con $1 \%$ de inulina aporta en la dieta $137 \mathrm{kcal}$. Si se tiene en cuenta que una porción de helado normal es de $80 \mathrm{~g}$, esta porción aportaría 109,6kcal y representaría el 5,48\%, de las 2000kcal recomendadas por la Organización Mundial de la Salud, en una dieta sana (FAO, 2015) (Tabla 2).

En la figura 1, se presenta un esquema que permite comparar el efecto de la adición de inulina sobre el contenido de grasa saturada y el aporte de calorías, en las diferentes formulaciones.

Al incorporar inulina en 1\% (reemplazando el 57,14\% de grasa vegetal), el contenido calórico decreció en 16\% y la grasa saturada disminuyó en 48,6\%.

Según la FAO (2003), la energía proveniente de las grasas no debería representar más del 30\% y la energía incluida en la ingesta diaria, proveniente de grasas saturadas, no debiera de exceder el 10\%, ya que incrementan el colesterol. Se conoce que el consumo de grasas saturadas aumenta el riesgo cardiovascular y que existen relaciones positivas posibles entre la ingesta de grasa saturada y el riesgo de padecer diabetes (FAO \& FINUT, 2012). Por lo tanto, se podría afirmar que el consumo de los helados de vainilla obtenidos en esta investigación, tendrían menor riesgo de sobrepeso para los consumidores, debido al menor aporte calórico. La capacidad que tiene la inulina de reemplazar la grasa vegetal en las formulaciones de helado de vainilla, se podría explicar por la capacidad que tiene la inulina para unir moléculas de agua y formar una red, incorporándose fácilmente con los otros ingredientes de la formulación (Franck, 2002). Las investigaciones consultadas de uso de inulina en helados no reportan el valor calórico, por lo cual, los resultados que aquí se presentan aportan información adicional de importancia en el mercadeo de productos bajos en calorías.

Evaluación sensorial: El ANOVA de las pruebas de aceptación mostró F mayor que el F crítico, lo que indica que los consumidores reconocieron diferencias entre los cuatro tratamientos. La tabla 3 presenta el ANOVA realizado a las respuestas de los catadores, quienes consumieron helado formulado con inulina.

En la tabla 4, se presentan los resultados de preferencia de los jueces consumidores.

Tabla 2. Contenidos de grasa saturada y aporte energético de helados de vainilla formulados con inulina comercial, como sustitutos de grasa y aporte de una porción de helado (basado en una dieta de 2000 kilocalorías/día).

\begin{tabular}{|c|c|c|c|c|}
\hline Tratamiento & E Kcal/100g & Grasa Sat \% & $\begin{array}{c}\text { E (Kcal/80g) } \\
\text { para una porción de } \\
\text { helado }\end{array}$ & $\begin{array}{c}\text { \% aporte } \\
\text { diario }\end{array}$ \\
\hline MI & $163 \pm 1,0$ & $3,7 \pm 0,2$ & $130,4 \pm 0,8$ & 6,52 \\
\hline M2 & $155 \pm 2,0$ & $3,4 \pm 0,5$ & $124 \pm 0,8$ & 6,2 \\
\hline M3 & $153 \pm 1,0$ & $3,1 \pm 0,4$ & $122,4 \pm 0,8$ & 6,12 \\
\hline M4 & $137 \pm 3,0$ & $1,9 \pm 0,4$ & $109,6 \pm 2,4$ & 5,48 \\
\hline
\end{tabular}




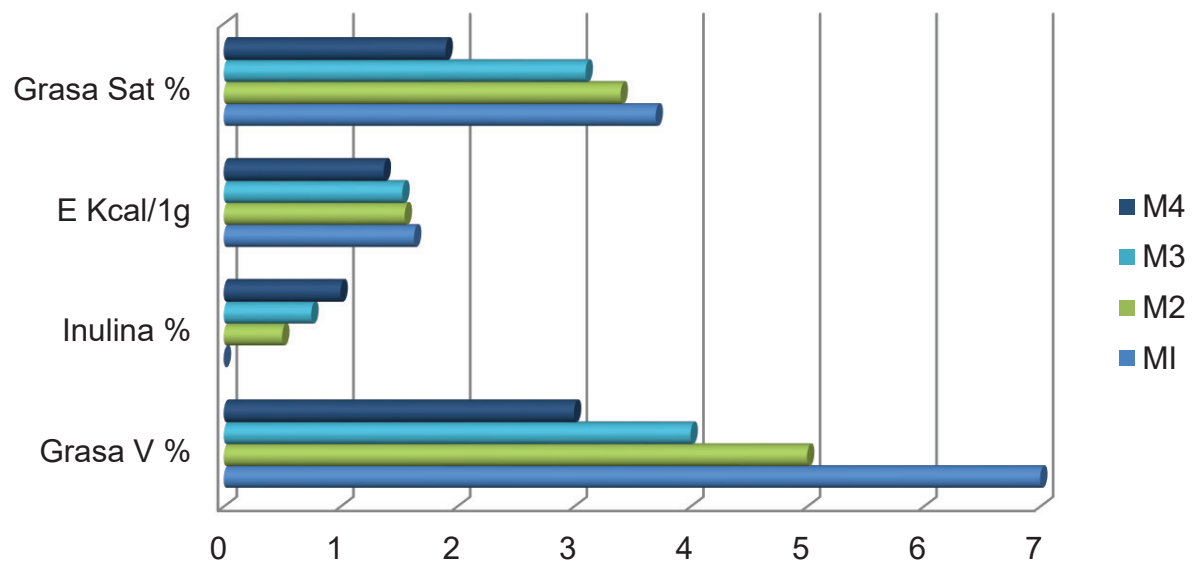

Figura 1. Gráfico que relaciona las variables de proceso y de respuesta para 4 formulaciones de helado de vainilla, que involucran inulina en diferentes porcentajes. Ver nomenclatura de los tratamientos en texto. Grasa v= grasa vegetal. Grasa sat = grasa saturada .

Tabla 3. Anova de prueba sensorial hedónica de siete puntos de helado de vainilla, formulado con inulina.

\begin{tabular}{|c|c|c|c|c|c|}
\hline & & & & & Relación $F$ \\
\hline Fuente de variación & g1 & SC & $\mathrm{CM}$ & Calculada & $\begin{array}{l}\text { Tabulada } \\
(\mathrm{p} \leq 0,05)\end{array}$ \\
\hline Total $(\mathrm{T})$ & 95 & 3391 & & & \\
\hline Tratamiento $(\mathrm{Tr})$ & 3 & 0,36 & 1130,33 & 0,002 & 3,03 \\
\hline Panelistas (p) & 23 & 31,24 & 1,36 & 0,0278 & 3,73 \\
\hline
\end{tabular}

Tabla 4. Distribución de frecuencias según preferencia de jueces consumidores de helado.

\begin{tabular}{|l|c|c|}
\hline \multicolumn{1}{|c|}{ Muestra } & Frecuencia & \%Preferencia \\
\hline M1 $=321$ & $\mathbf{7}$ & 29,2 \\
\hline M2=768 & 3 & 12,5 \\
\hline M3 $=098$ & 5 & 20,8 \\
\hline M4=432 & 9 & 37,5 \\
\hline Total & $\mathbf{2 4}$ & $\mathbf{1 0 0}$ \\
\hline
\end{tabular}

No se presentaron diferencias estadísticamente significativas en las variables de respuesta entre los tratamientos ( $\mathrm{p} \geq 0,05$ ), lo que significa que (M1), (M2), (M3) y (M4) tuvieron el mismo grado de aceptación y que el helado, a pesar de tener menor contenido graso, conservó las características de la muestra de referencia (M1).

Durante la evaluación sensorial, se realizó una prueba de preferencia, con el objetivo de que los panelistas escogieran una muestra como la preferida entre 4 pruebas; la testigo, sin adición de inulina y las muestras que contenían el 0,5, el 0,75 y el 1\%. El tratamiento menos preferido fue donde se utilizó el $0,5 \%$ de inulina en la receta $(12,5 \%$ de preferencia), comparada con la muestra testigo (29,2\% de preferencia). El tratamiento más preferido fue donde se utilizó el $1 \%$ de inulina $(37,5 \%$ de preferencia).

La información contrastada con los resultados obtenidos de la prueba hedónica da bases para afirmar que la adición de inulina hasta el $1 \%$ en las recetas no confiere cambios sensoriales significativos y los panelistas prefieren el producto que tiene el 1\% de inulina, sobre los demás tratamientos.

La inulina ha sido utilizada para reformular productos, con el fin de disminuir el contenido de grasa en las formulaciones. Estudios reportados por Ismail et al. (2013), indican que la adición 
de inulina en helados, desde el 2,5 hasta el $5 \%$ en la mezcla, modifican las características sensoriales en el producto terminando, incrementándose la dureza, la adhesividad, la masticabilidad, la gomosidad en el producto, características que el consumidor puede percibir con facilidad; sin embargo, en el presente trabajo no se midieron dichas propiedades. Los ensayos realizados por este grupo de investigadores, con niveles menores de inulina en la mezcla hasta el 1\%, no fueron detectados por los consumidores y lograron disminuir el valor calórico del producto (Ismail et al. 2013). Akbari et al. (2016) incorporaron 2, 3, y 4\% de inulina en formulaciones de helado, obteniendo similitudes en los análisis sensoriales en los helados formulados con $2 \%$ de inulina y Rodríguez et al. (2015), incluyeron inulina y almidón modificado en la formulación de helados y obtuvieron una formulación con características sensoriales semejantes a la muestra control.

En la literatura consultada no se encontraron reportes científicos provenientes de Colombia donde se utilice inulina como mimético de grasa en helados, por lo cual, esta investigación abre las puertas a la empresa Meals de Colombia para la oferta de helados bajos en calorías, de gran aceptación sensorial. Además, con la oferta de helados bajos en calorías, la empresa Meals de Colombia podría cumplir con la responsabilidad social que tienen las empresas de contribuir a disminuir un problema de salud pública en Colombia, como lo es el sobrepeso y la obesidad.

De esta investigación, se puede concluir que la inclusión de inulina en la formulación de helados podría ser una estrategia para ofertar productos de alto consumo, con menor riesgo de obesidad. En la formulación de helados de vainilla es posible reemplazar el $57,14 \%$ de grasa vegetal por inulina, sin que se afecten propiedades sensoriales en el producto, logrando reducir en 16\% del contenido calórico y 48,6\% el contenido de grasa saturada. Se requieren otras investigaciones, que permitan definir la cantidad máxima de inulina, que se puede adicionar a una formulación de helado sin que ocasione pérdidas de palatabilidad. Además, se requiere conocer si con los niveles de inulina adicionados a la formulación de helado, se podrían declarar en el producto, el Claim de prebiótico.

Conflicto de Intereses: El manuscrito fue preparado y revisado con la participación de todos los autores, quienes declaramos que no existe ningún conflicto de intereses que ponga en riesgo la validez de los resultados presentados. Financiación: Esta investigación fue financiada por Meals de Colombia S.A.S y por la Universidad Nacional de Colombia Sede Palmira.

\section{REFERENCIAS}

1. AKALIN, A.S.; KARAGÖZLÜ, C.; ÜNAL, G. 2008. Rheological properties of reduced-fat and low-fat ice cream containing whey protein isolate and inulin. Eur. Food Res. Technol. 227:889-895. https://doi.org/10.1007/s00217007-0800-z

2. AKBARI, M.; HADI, M.; NIAKOSARI, M.; BEDELTAVANA, A. 2016. The effect of inulin on the physicochemical properties and sensory attributes of low-fat ice cream. Int. Dairy J. 57:52-55. https://doi.org/10.1016/j. idairyj.2016.02.040

3. AKBARIA, M.; ESKANDARIB, M.H.; DAVOUDIC, Z. 2019. Application and functions of fat replacers in low-fat ice cream: A review. Trends Food Sci Technol. 86:34-40. https://doi.org/10.1016/j.tifs.2019.02.036

4. AOAC International. 2001. Official method 996.06: Fat (Total, saturated and unsaturated) in food. Rockville, MD. Disponible desde Internet en: http://files.instrument. com.cn/bbs/upfile/2008622221856.pdf (con acceso el 01/06/2019)

5. BARCLAY, T.; GINIC-MARKOVIC, M.; JOHNSTON, M.R.; COOPER, P.D.; PETROVSKY, N. 2012. Analysis of the hydrolysis of inulin using real time $1 \mathrm{H}$ NMR spectroscopy. Carbohydr. Res. 352:117-125. https://doi.org/10.1016/j. carres.2012.03.001

6. BAYARRI, S.; CHULIÁ, I.; COSTELL, E. 2010. Comparing $\lambda$ - carrageenan and an inulin blend as fat replacers in carboxymethyl cellulose dairy desserts. Rheological and sensory aspects. Food Hydrocoll. 24(6):578-587. https:// doi.org/10.1016/j.foodhyd.2010.02.004

7. DOOLEY, L.; LEE, Y.-S.; MEULLENET, J.-F. 2010. The application of check-all-that-apply (CATA) consumer profiling to preference mapping of vanilla ice cream and its comparison to classical external preference mapping. Food Qual. Prefer. 21:394-401. https://doi.org/10.1016/j. foodqual.2009.10.002

8. ENSIN. 2015. Encuesta nacional de la situación nutricional. Ministerio de Salud y Protección Social, Instituto Nacional de Salud (INS), Departamento Administrativo para la Prosperidad Social, Instituto Colombiano de Bienestar Familiar (ICBF), Universidad Nacional de Colombia. Colombia. Disponible desde Internet en: Www.minsalud. gov.co/sites/rid/Lists/BibliotecaDigital/RIDE/VS/ED/ GCFI/ensin-colombia-2018.pdf (con acceso 08/07/2019)

9. FRANCK, A. 2002. Technological functionality of inulin and oligofructose. British J. Nutr. 2: 287-291. https://doi. org/10.1079/BJN/2002550

10. GOFF, H.D.; HARTEL, R.W. 2013. Ice cream (7th ed.). New York: Springer. 462p.

11. GUINARD, J.X.; ZOUMAS-MORSE, C.; MORI, L.; PANYAM, D.; KILARA, A. 1996. Effect of sugar and fat on the acceptability of vanilla ice cream. J. Dairy Sci. 79(11):1922-1927. https://doi.org/10.3168/jds.S00220302(96)76561-X 
12. HERNÁNDEZ-CARRANZA, P.; JIMÉNEZ-MUNGUÍA, M.T. 2010. Propiedades funcionales y aplicaciones industriales de los fructo-oligosacáridos. TSIA. 4(1):1-8.

13. ICONTEC. 2002. Norma Técnica Colombiana 1239 (Segunda actualización). Helados y Mezclas para helados.18p.

14. INVIMA. 1989. Ministerio de Salud "Resolución 1804 de 1989". p.1-9.

15. ISMAIL, E.A.; AL-SALEH, A.A.; METWALLI, A.A.M. 2013. Effect of inuline supplementation on rheological properties of low-fat ice cream. Life Sci. J. 10(3):1742-1746.

16. KOLIDA, S.; GIBSON, G.R. 2007. Prebiotic capacity of inulintype fructans. J. Nutr. 137(11suppl.):2503S-2506S. https:/ / doi.org/10.1093/jn/137.11.2503S

17. LI, W.; ZHANG, J.; YU, C.; LI, Q.; DONG, F.; WANG, G.; GUO, Z. 2015. Extraction, degree of polymerization determination and prebiotic effect evaluation of inulin from Jerusalem artichoke. Carbohydr. Polym. 121:315-319. https:/ / doi.org/10.1016/j.carbpol.2014.12.055

18. LIU, R.; WANG, L.; LIU, Y.; WU, T.; ZHANG, M. 2018. Fabricating soy protein hydrolysate/xanthan gum as fat replacer in ice cream by combined enzymatic and heatshearing treatment. Food Hydrocoll. 81:39-47. https:// doi.org/10.1016/j.foodhyd.2018.01.031

19. MARSHALL, R.T.; GOV, H.D.; HARTEL, R.W. 2003. Ice cream. 6th ed. Kluwer/Plenum Publishers, New York. 195 p.

20. MENSINK, M.A.; FRIJLINK, H.W.; VAN DER VOORT MAARSCHALK, K.; HINRICHS, W.L.J. 2015. Inulin, a flexible oligosaccharide I: Review of its physicochemical characteristics. Carbohydr. Polym. 130:405-419. https:// doi.org/10.1016/j.carbpol.2015.05.026

21. ORGANIZACIÓN DE LAS NACIONES UNIDAS PARA LA ALIMENTACIÓN Y LA AGRICULTURA, FAO. 2003. La OMS y la FAO publican un informe de expertos independientes sobre dieta, nutrición y prevención de enfermedades crónicas. En Comunicados de prensa. Disponible desde Internet en: http://www.who.int/ mediacentre/news/releases/2003/pr20/es/ (con acceso 11/12/2017).

22. ORGANIZACIÓN DE LAS NACIONES UNIDAS PARA LA ALIMENTACIÓN Y LA AGRICULTURA, FAO. 2015. Alimentación sana. Disponible desde Internet en: http://www.who.int/mediacentre/factsheets/fs394/es/ (con acceso 11/12/2017).
23. ORGANIZACIÓN DE LAS NACIONES UNIDAS PARA LA ALIMENTACIÓN Y LA AGRICULTURA, FAO; FUNDACIÓN IBEROAMERICANA DE NUTRICIÓN, FINUT. 2012. Grasas y ácidos grasos en nutrición humana. Granada, España Consulta de expertos, Paper No. 91, p.1204.

24. PORTAFOLIO. 2019. Colombia: Así se mueve la industria de los helados en el país. Disponible desde Internet en: https://www.portafolio.co/negocios/helados-unaindustria-que-seduce-a-grandes-y-chicos-526211 (con acceso 02/07/2019).

25. RODRÍGUEZ, L.; CAMPDERRÓS, M.E. 2015. Incorporación de inulina en la matriz de helado de vainilla libre de grasa: efecto sobre las propiedades texturales y físicas. La Alimentación Latinoamericana. 316:66-69.

26. THOMPSON, K.R.; CHAMBERS, D.H.; CHAMBERS, E. 2009. Sensory characteristics of ice cream produced in the U.S.A. and Italy. J. Sens Stud. 24:396-414. https://doi. org/10.1111/j.1745-459X.2009.00217.x

27. TIWARI, A.; SHARMA, H.K.; KUMAR, N.; KAUR, M. 2015. The effect of inulin as a fat replacer on the quality of lowfat ice cream. Internal J. Dairy Technology. 68(3):374-380. https://doi.org/10.1111/1471-0307.12176

28. UNILEVER, Holanda. 2016. Información Nutricional. Disponible desde Internet en: https://www.unilever. com.mx/Images/informacio-n-nutrimental---holanda_ tcm1286-497428_1_es.pdf, (con acceso 19/11/2017).

29. YAZICI, F; AKGUN, A. 2004. Effect of some protein based fat replacers on physical, chemical, textural, and sensory properties of strained yoghurt. J. Food Engineering. 62:245254. https://doi.org/10.1016/S0260-8774(03)00237-1

30. ZOU, M.L.; MOUGHAN, P.J.; AWATI, A.; LIVESEY, G. 2007. Accuracy of the Atwater factors and related food energy conversion factors with low-fat, high-fiber diets when energy intake is reduced spontaneously. Am. J. Clin. Nutr. 86(6):1649-1656. https://doi.org/10.1093/ajcn/86.5.1649

31. ZOULIAS, E.I.; OREOPOULOU, V.; TZIA, C. 2002. Textural properties of low-fat cookies containing carbohydrate- or protein-based fat replacers. J. Food Engineering. 55:337342. https://doi.org/10.1016/S0260-8774(02)00111-5 